\title{
Trabajo social en los nuevos escenarios de infancia, adolescencia y familia $^{1}$
}

\author{
Ángela María Quintero Velásquez*
}

\begin{abstract}
RESUMEN
El Trabajo Social históricamente está vinculado con metodologías de intervención, que son exigidas y resaltadas por las nuevas leyes. El artículo reseña la fundamentación teórica y las estrategias metodológicas de uso obligatorio o discrecional, que contribuyen al papel del peritaje social y del informe social, en contextos multidisciplinarios, que son rastreadas terminológicamente y analizadas en la investigación: Pruebas Psicosociales en Derecho de Infancia, Familia y Adolescencia.
\end{abstract}

Palabras clave: Trabajo Social / Familia / Niñez / Infancia / Adolescencia / Multidisciplinariedad / Derechos.

\section{Trabalho social na etapa nova de meninos, adolescents e famílias}

\begin{abstract}
O Trabalho Social historicamente está vinculado com metodologías de intervenção, que são exigidas e ressaltadas pelas novas leis. $O$ artigo reseña a fundamentación teórica e as estratégias metodológicas de uso obrigatório ou discrecional, que contribuem ao papel do peritazgo social e do relatório social, em contextos multidisciplinarios, que são rastreadas terminológicamente e analisadas na investigação Provas Psicosociales em Direito de Infância, Família e Adolescencia.
\end{abstract}

Palavras chave: Trabalho Social / Família / Niñez / Infância / Adolescencia / Multidisciplinariedad / Direitos.

\section{Social work in the new stage of children, adolescents and families}

\section{ABSTRACT}

Historically, the Social Work is linked to intervention methodologies which are now required by the new laws, especially concerning to psychosocial

1 Artículo basado en la ponencia del mismo título, presentada en XIX Seminario Latinoamericano de Escuelas de Trabajo Social, Universidad Católica de Santiago de Guayaquil ALEITS, 2009. Es derivado de la investigación Pruebas Psicosociales en Derecho de Infancia, Adolescencia y Familia (2009-10). Universidad de Antioquia. Centro de Investigaciones Jurídicas. Área Derecho Familia. Medellín. Colombia.

* Colombiana. Trabajadora Social. Magíster en Educación, Orientación y Consejería. Docente del Departamento de Trabajo Social. Universidad de Antioquia, Colombia. Correo electrónico: jesus@epm.net.co 
tests. The article reviews the theoretical and methodological strategies of mandatory or discretionary use, which contribute to the role of specialist social report, and social report in multidisciplinary contexts, which are terminologically tracked and analyzed in Psychosocial Tests in Child, Family and Adolescence Law Research.

Key words: Social work / family / childhood/infancy / adolescence / multidisciplinarity / rights. 


\section{Introducción}

La Universidad de Antioquia, Medellín a través de la Facultad de Derecho y Ciencias Políticas y el Departamento de Trabajo Social, agrupados en el Área de Derecho de Familia, desarrolla desde hace dos décadas procesos conducentes a la convergencia de los saberes y a fortalecer los enlaces socio-jurídicos propios del Enfoque en los Derechos y la prioridad en la Niñez, Adolescencia y Familia, como actores políticos y sujetos prevalentes. En la línea de investigación universitaria realizan el análisis de las Pruebas Especiales, contempladas en la Ley 1098 de noviembre de 2006, Código de Infancia y Adolescencia

El artículo sustenta la colaboración multidisciplinaria y algunos de los conceptos más significativos, propios del Trabajo Social en su repertorio profesional, que hoy son relevantes en estas nuevas disposiciones jurídicas.

La investigación es de carácter multidisciplinario, con la Convergencia de las Ciencias Jurídicas (Derecho), Sociales (Trabajo Social y Psicología) y de la Información (Terminología y Traducción). Entre sus objetivos, está:

- Analizar en contextos escritos, el alcance de los estudios psicosociales en el campo del Derecho de Infancia, Adolescencia y Familia.

- Fundamentar la importancia de las Pruebas especiales en Derecho de Infancia, Adolescencia y Familia, por medio de entrevistas a expertos.

- Realizar el rastreo terminológico y la validación del uso de los términos: prueba, concepto, peritaje, dictamen e informe, entre otros.

Bajo los criterios de la investigación cualitativa, se trata de un estudio documental, de tipo exploratorio, apoyado fundamentalmente en las fuentes secundarias de información y en el análisis conceptual

El carácter de investigación hermenéutica - documental está dado por el rastreo bibliográfico y terminológico de los vocablos que aplican los especialistas en el tema de las pruebas especiales de infancia, adolescencia y familia, en el marco de la Constitución Política de 1991 y en la Ley 1098 de 2006 de Colombia. Sobre esa búsqueda se establecerá la diferencia conceptual y analizará la 
polisemia ${ }^{2}$ en el uso de los términos: prueba, concepto, peritazgo, dictamen e informe, con el fin de su validación.

El presente artículo reseña la importancia y la función profesional de Trabajo Social en el sistema jurídico, focalizado en niñez, adolescencia y familia. Lo que es contemplado en el Código de Infancia y Adolescencia

-Ley 1098 de 2006-, con el fin de cumplir un papel relevante en la administración de la justicia y de las pruebas psicosociales. Documentación y experiencia que es base de la investigación, en la recolección y procesamiento de la información.

El énfasis documental centrará el examen de la producción científico-técnica sobre el campo socio-judicial durante la última década, de las disciplinas delimitadas. La investigación está en ejecución, hasta agosto 2010, en la ciudad de MedellínColombia.

\section{Aportes disciplinarios}

La modernización de los Estados y la suscripción de estos a los convenios y tratados internacionales sobre la educación, la promoción, la protección y la defensa de los derechos fundamentales, establece nuevas rutas para la formación universitaria y para los procesos de atención integral, en una de las áreas más significativas para el Trabajo Social contemporáneo: los sistemas humanos.

Relevando a la familia como sujeto prevalente de derechos (proponiendo también que sea prevalente en los procesos administrativos y judiciales), y como sujeto político, con base en las relaciones ecosistémicas, en los desarrollos alternativos (sustentable y a escala humana), y en las nociones modernas de la ciudadanía y la ética civil. Es necesario que los cambios sociojurídicos que impactan los marcos legislativos y constitucionales, sean asimilados y compartidos de manera colaborativa por todas las áreas del conocimiento.

En el proceso de formación integral, compete a las unidades académicas y centros de estudios superiores, configurar

2 Pluralidad de significados de una palabra o de cualquier signo lingüístico. 2. Pluralidad de significados de un mensaje, con independencia de la naturaleza de los signos que lo constituyen. 3. Relación entre las designaciones y los conceptos en una lengua en la que una designación representa dos o más conceptos. (Quintero, 2007: 99) 
alternativas pedagógicas, didácticas, cognitivas e incluyentes, que faciliten que el profesional de Trabajo Social coloque todo su repertorio conceptual y metodológico en el contexto de las exigencias constitucionales y legislativas de cada país y del continente, para responder a los requerimientos en términos de peritazgo social, estudio socio-familiar, mediación y conciliación familiar, acogimiento familiar, custodia compartida, movilidad social (migraciones internas y transnacionales, desplazamiento forzado).

Es necesario mirar el alcance que hoy tiene la prueba pericial en los países de la región, siendo fundamental está prueba en asuntos como adopciones, custodia y cuidado personal, reglamentación de visitas, interdicciones, entre otros; lo que obliga a la comunidad académica a procurar un acercamiento a los dictámenes rendidos por psicólogos, trabajadores sociales, nutricionistas y todos aquellos profesionales que con su experiencia y conocimiento son auxiliares de una pronta y eficaz justicia.

El Trabajo Social incorpora sus destrezas y habilidades centenarias (en tanto profesión de cien años), con las fortalezas propias de las tendencias y enfoques contemporáneos, donde la profesión se ha destacado con solvencia teórica y práctica, en los contextos de intervención con familias y sus miembros. Como operador social en la rama judicial y afines, es un profesional responsable del dictamen en procesos vitales, que comprometen el proyecto vital de las personas, pues le corresponder aportar en el diagnóstico sobre alimentos, patria potestad, visitas, violencia familiar, abuso sexual y maltrato infantil, justicia restaurativa, entre otros.

Todo lo anterior exige una formación universitaria polivalente, creativa, donde predomine la expansión del conocimiento, la capacidad investigativa, la cooperación de saberes, las alianzas multisectoriales e interinstitucionales, las teorías jurídicas y constitucionales, para potenciar en los nuevos escenarios legislativos, la compleja realidad, estudiar e incorporar en su acervo, el análisis del contexto en términos de los fenómenos más cercanos a la práctica profesional: pobreza, indigencia, exclusión, multiculturalismo, diversidad en sus diferentes órdenes.

Los enlaces socio-jurídicos, mediados por la promulgación de nuevas o reformadas leyes, para atender, proteger y promover a la familia como un todo o a sus miembros, permite el crecimiento exponencial de las ciencias sociales en el campo jurídico. Derivando en el imperativo de asumir un protagonismo 
profesional a través del concepto especializado que emitan los profesionales acreditados, en los procesos judiciales. Tal como ocurre en la mediación, la conciliación y el peritazgo social, que emergen en gran parte de las legislaciones recientes de los países de la región.

Para tal efecto, el Trabajo Social en particular, aporta sus estrategias y procedimientos disciplinarios ya convalidados, en conjunción con las alianzas del pensamiento y la cooperación entre las áreas del conocimiento, propendiendo lenguaje especializado y el análisis ecosistémico. El tema específico de niñez, infancia, adolescencia y familia, se constituye en ámbito de potestad profesional, refrendado por las disposiciones legales que acreditan este ejercicio.

En lo referido a los códigos y preceptos derivados del acatamiento a la normativa internacional de infancia, resaltan las contribuciones conceptuales y metodológicas retomadas para garantizar el cumplimiento y restablecimiento de los derechos, entre ellos el de tener una familia y de la debida atención del Estado a su desarrollo y satisfacción de sus condiciones vitales: La sociedad, y de modo particular el Estado y las Organizaciones Internacionales, deben proteger la familia con medidas de carácter político, económico, social y jurídico, que contribuyan a consolidar la unidad y la estabilidad de la familia para que pueda cumplir su función específica (Carta de los Derechos de la Familia, preámbulo I, 1983).

Cumpliendo el mandato emanado de la Convención Internacional de los Niños, Colombia reforma la legislación y en noviembre de 2006, con la Ley 1098, adopta el Nuevo Código de la Infancia y la Adolescencia. Ratifica la consideración universal de que la Familia y la Niñez son sujetos de derechos y promulga el principio de la corresponsabilidad, la participación mancomunada entre Estado - Familia - Sociedad Civil. Entre otras normas, establece de manera obligatoria que el profesional de Trabajo Social debe operar en las Comisarías de Familia (creadas en 1989) y el sistema judicial (juzgados de Familia, de menores, promiscuos, de ejecución de penas y medidas de seguridad), con perfiles que enfatizan el peritazgo social y el trabajo multidisciplinario, bajo la denominación de equipos psico-sociales.

Esta ordenanza legal no aplica en todas las instituciones jurídicas y no existen procedimientos claros para cumplir, dependiendo en gran medida del criterio del Juez y de la administración 
municipal (alcaldes o concejos). La figura del cargo de asistentes sociales que faculta a varios profesionales de las ciencias sociales y humanas, es obligatoria por la ley en mención, y coloca al Trabajo Social en la competencia de capacitarse con sus estrategias y fundamentación teórica, en la aplicación de los nuevos dispositivos legales que le asignan potestad para que sus evaluaciones y valoraciones diagnósticas sean reconocidas e incorporadas en todos los procesos. Uno de los aspectos más novedosos de la ley es la instauración de un sistema penal que garantice los derechos de los jóvenes infractores y de la oralidad, como elementos de la llamada justicia restaurativa, y allí el Trabajo Social ha comprometido su desempeño técnico.

Las formas alternativas de enfrentar los conflictos sociofamiliares, ante todo la conciliación (Equidad y en Derecho) y la Mediación, son propios del ejercicio profesional y son ejercidos con fluidez en distintos ámbitos (familiar, laboral, comercial, educativo, comunitario), dada la preparación básica en la formación universitaria y las destrezas inherentes al perfil. La figura de los Jueces de Paz, Casas de la Justicia y otros dispositivos de la legislación colombiana, facilitan también la participación activa del Trabajo Social con los sistemas humanos ${ }^{3}$. Cumple además un papel destacado en la sensibilización de funcionarios públicos y de las comunidades para la observancia de la norma y amplía su espectro hacia las políticas y programas de atención integral de primera infancia, violencia familiar y seguridad alimentaria.

El Trabajo Social y el pensamiento sistémico contribuyen con el análisis de la perspectiva evolutiva, los Ciclos Vitales ${ }^{4}$, para entender en profundidad las necesidades y exigencias de estas etapas fundamentales en el ser humano, niñez, infancia y adolescencia, que el legislador maneja con criterios que no siempre se ajustan a las teorías del desarrollo de Piaget, Freud y Ericsson. Ellas sustentan los títulos de primera infancia, tan en boga en los códigos y políticas públicas, aunque no siempre corresponden a las características etarias. Ilustra también los requerimientos esenciales de la adolescencia como fase de reajuste de la jerarquía y del statu - quo, agravado por las deficientes condiciones de vida, la angustia existencial y el sentido de supervivencia en

3 Entiéndase: individuos, familias, grupos, comunidades, corporaciones, que en esencia están constituidas por personas.

4 Anclados en la teoría del Desarrollo y en la psicología evolutiva, están el ciclo individual, el familiar y el de pareja, asumida como un proceso en interrelación de todas las fases. 
situaciones extremas de vulneración de los derechos mínimos y ausencia de la debida protección psico-afectiva.

Merece también incluirse la conceptualización sobre familias multiproblemáticas, ${ }^{5}$ que presentan características que compaginan con la mayoría de los niños, las niñas y los adolescentes que forman parte activa del sistema judicial y como tal, revelan otras dinámicas y requieren análisis transversales y longitudinales, para comprender y asumir los riesgos en su protección. En otro sentido, son familias con crisis estructurales, porcuanto reportan permanentemente situaciones disfuncionales o conflictos que se expresan a través de sus miembros en tempranas edades.

Ello exige que los profesionales, instituciones y poderes gubernamentales, en general todos los operadores sociales y jurídicos, reconozcan esta fundamentación científica y la incorporen en los programas de atención integral, donde son convocados expertos de diferentes ramas, que coadyuvan en el dictamen.

De igual manera se reconoce el valor que tienen los trabajadores sociales como el profesional más indicado para estudiar y valorar el ambiente en que se encuentra inmerso el individuo y también sus proyecciones futuras y propuestas de mejora, lo cual puede ser utilizado en el ámbito judicial en caso necesario. Los trabajadores sociales que actúan como peritos se encargan de emitir todos los dictámenes periciales que están relacionados con la situación de las personas, su entorno próximo y sus redes sociales, con la finalidad de informar al juez y a todos aquellos que requieran su intervención (Quintero, 2009:8).

La evaluación socio-familiar es un distintivo disciplinario de la práctica en contextos no clínicos -donde se desarrollan las acciones jurídicas-, que abarca diversas estrategias: visita domiciliaria, observación, entrevista individual o familiar, remisión a recursos, coordinación interinstitucional, intersectorial y multidisciplinaria, entre otras. La innovación, que se plantea con los procedimientos recientes, está dada porque el perfil profesional sea hábil y creativo, trascendiendo la aplicación de la técnica por la técnica, para, parafraseando a Rosa María Cifuentes (2008), contrarrestar la actual tendencia instrumental. Superar la perplejidad y ambigüedad ante la compleja situación actual;

$5 \quad$ Para ampliar el concepto VER: Coletti y Linares (1997), Valentín González (2002). 
interactuar con otras disciplinas y profesiones para construir conjuntamente alternativas de desarrollo social en y para América Latina.

Dado que los aparatos gubernamentales y judiciales no disponen aún de los espacios y condiciones logísticas, pertinentes para garantizar la intimidad, las erupciones pasionales y la subjetividad de las personas atendidas, se requiere la optimización de los dominios estratégicos y técnicos, para procurar una atención integral. Para tal efecto el asumir la conciliación, la mediación, la justicia restaurativa, los grupos socio-educativos y socioterapéuticos, la intervención en redes, la activación de la resiliencia, entre otros, favorecería la inclusión de gran parte de los factores que determinan la situación y devuelve a los sistemas atendidos, sus recursos internos para enfrentar la acción jurídica.

La cosmovisión profesional y corporativa que trascienda el alcance punitivo o la sanción penal, favorece que el alcance de las leyes del menor, de infancia, niñez, adolescencia o juventud -según las diferentes denominaciones continentales-, no reproduzca esquemas de exclusión, inequidad, violencia, mortandad y pobreza. Comprometer a las comunidades a través de las redes informales o primarias, en la reparación del daño, más que en el confinamiento a instituciones con evidentes limitaciones en su capacidad presupuestal y en la respuesta oportuna y rápida de las complejas situaciones de los niños, las niñas y los adolescentes que viven en situación de vulnerabilidad, acogería parte de las disposiciones del desarrollo sustentable y humano, aporta a la promoción y defensa de la autonomía y los intereses locales, contribuye en el proceso formativo y rompe el ciclo determinista.

Es factible idear acciones socioeducativas adecuadas y dimensionadas al delito o la falta cometida por el niño, la niña o el adolescente, valorando sus circunstancias con el dictamen social y así facilitar la reinserción y evitar la criminalización. De igual manera la mediación familiar y comunitaria es necesaria para alcanzar acuerdos extrajudiciales que eviten el internamiento. No basta con que la norma legal diferencie las edades para aplicar sanciones y exonerar de responsabilidades a los menores de catorce años, sino que se requieren cambios relacionados con la pericia técnica interdisciplinaria, para que cumpla su cometido de análisis integral y colaborativo del entorno. 
Esteejercicio requiere una apropiadayponderada conjunción entre la instrumentalidad, necesaria e implícita en nuestro quehacer disciplinario -que incluso es copiada por otras disciplinas, que han revaluado sus prácticas para acceder a posturas menos dogmáticas y jerárquicas, y aceptar sus posibilidades de realizar intervención en contextos no cínicos-, la ética y la producción de saberes. La acción profesional no puede reducirse a la aplicación de la técnica o el acto operativo.

La tecnología y la técnica son vehículos para desplegar capacidades y aprender a ser, conocer, hacer, convivir. Es necesario ver si son justas o no, si respetan la integridad, la dignidad y la autonomía de las personas; si sus consecuencias son positivas o negativas, saber quién paga los costos y quién recibe los réditos de su aplicación. En síntesis, ¿qué criterios éticos se proponen e implementan para producir buenos conocimientos y acciones hacia la sociedad? (Melano, 2006:52).

\section{Retos del Trabajo Social en el campo judicial}

Este panorama legislativo requiere de un profesional de trabajo social, formado en el nuevo paradigma del Derecho -más allá del discurso ideológico sobre los Derechos Humanos, que de tanto usarlo en tramas vacías, pierde la relevancia y el análisis de contexto- para trascender la sola denominación y connotarlo desde la ética civil y profesional, en coherencia entre la palabra y las prácticas personales y ciudadanas.

Conceptos como justicia restaurativa, mediación, conciliación, convergencia, requieren competencias disciplinarias que integren los procedimientos clásicos, con los nuevos desafíos y el cambio de época. Esto es reconocer que coexisten los conocimientos y las metodologías tradicionales, con las contemporáneas y mas allá de poseer o adquirir información acumulada -fácilmente accesible con un "clic" en el computador, sin importar si responde o no, a documentación científica o arbitrada-, se trata de una cambio de cosmovisión, de expandir las fronteras intelectuales y cognitivas y abrirse a otros universos y percepciones, diferentes a la subjetividad u opinión personal.

Varios de los planteamientos derivados de la no agotada discusión sobre la modernidad y la postmodernidad, focalizan a la Familia 
como el principal sistema social, integral, único y complejo en permanente transformación donde prima su carácter relacional, relevando la importancia de su función socializadora primaria y las relaciones ecosistémicas. De esta manera, las reformas legales y constitucionales recientes en la región, tienden a reconocer otras variables, diferentes a la estructura y composición familiar, para estudiar, analizar e intervenir con la familia y sus miembros.

"Se trata de procesos que introducen un conjunto de desafíos para las profesiones que actúan en el campo socio-judicial, principalmente si se considera que lo hacen en un contexto caracterizado también por la individualización de lo social (Beck 1998), y, en consecuencia, por la progresiva judicialización de las expresiones de la cuestión social. En ese proceso, el desarrollo de nuevos dispositivos como la mediación y el arbitraje crean también nuevos territorios para dirimir conflictos sociales, al tiempo que amplían o redefinen, según los casos, las competencias de algunas profesiones del campo socio-judicial, entre otras, del Trabajo Social.

Ese tipo de procesos se traduce en la emergencia de nuevas influencias políticas, ideológicas y técnico-burocráticas sobre una profesión cuyo ejercicio se caracteriza por una inscripción estatal, asalariada y jerárquicamente dependiente de otras profesiones y campos de conocimiento entre los cuales se destaca el derecho. Sin embargo, como ocurre con todas las profesiones, el Trabajo Social posee -al menos potencialmente- capacidad para construirse y reconstruirse, a medida que eso mismo ocurre con la sociedad de la cual forma parte (Freidson 1988). Debido a ello, el surgimiento de nuevos problemas sociales, así como la emergencia de nuevas modalidades de gestión de los mismos, establecen un conjunto de límites estructurales y funcionales para el ejercicio de las profesiones en general y del Trabajo Social en particular." (De Martino, et al. 2008: 149).

Los enfoques contemporáneos centrados en los Derechos Humanos, según la Convención Internacional de los Derechos de la Niñez, y la ONU, establecen como prioritarias las acciones estatales y jurídicas, que amparen la no vulneración y el restablecimiento de los derechos de los niños, las niñas o los adolescentes. Los países deben aplicar la normativa internacional en el área de infancia y adolescencia, crear en el Poder Judicial los equipos de asesoramiento a los Juzgados de Familia 
Especializados y a los Juzgados de Adolescentes ${ }^{6}$ y modificar en el uso del lenguaje especializado y de las prácticas evaluativas, la comprensión de los procesos intra y extrafamiliares, para asumir posturas multidisciplinarias y de colaboración entre las áreas del conocimiento.

\section{Conclusiones}

La corresponsabilidad -entre familia - estado - sociedad- como vocablo reciente en la legislación, es reconocida de vieja data en el Enfoque de Convergencia, y permite generar un encuentro entre el sector social y el gobierno, para contribuir al análisis con visión propositiva, de los temas transversales del desarrollo social, niñez, juventud, infancia, de tal forma que se planteen elementos de concertación para el diseño, la ejecución y la evaluación de las políticas públicas sociales.

Las innovaciones estatales y normativas contemporáneas son un excelente escenario para fortalecer el papel clásico del Trabajo Social, en los Contextos no Clínicos: recepción, orientación, evaluación, prevención, promoción, educación, formas alternativas de enfrentar los conflictos socio-familiares. Es la oportunidad histórica de participar de manera más decisiva y profesional, en la aplicación e interpretación de las leyes, en la garantía y restablecimiento de los derechos fundamentales y en la atención integral a los sectores más desprotegidos de la sociedad: la niñez y la familia en condiciones de exclusión, inequidad, pobreza e indigencia.

Los fundamentos del pensamiento sistémico que posibilitan el cambio en las relaciones humanas, facilitan la co-creación y la colaboración de las áreas del conocimiento facultadas para ser operadores socio-jurídicos. Esto determina desafíos para las unidades de formación universitaria, particularmente las ciencias sociales, en el desarrollo de habilidades de trabajo multidisciplinario, capacidad de operar con ámbitos inciertos y ambiguos, una sólida fundamentación jurídica, el fortalecimiento de las técnicas disciplinarias e investigativas, la interacción en redes virtuales y presenciales y la producción de conocimiento a partir de la investigación básica.

6 Las títulos de los juzgados o corporaciones varían en cada país; en esencia responden al interés mundial de asignarle nombre propio a las competencias jurídicas, de niñez, adolescencia y familia. 
Para la ilustración, se enuncia la importancia de la Red Asistentes Sociales Jurídicos de Colombia ${ }^{7}$ : A la luz de la modernización del Estado y las nuevas disposiciones jurídicas, la trabajadora social Carmen Tulia Cuadros Restrepo (Medellín) crea en el 2006 este dominio de alcance iberoamericano, para compartir discusiones académicas, construir procesos de identidad disciplinaria y fomentar la multidisciplinariedad y la intersectorialidad. Colocando el ciber - espacio a tono con las reformas jurídicas, especialmente en Niñez, Adolescencia y Familia, donde los profesionales de Trabajo Social tienen un mandato constitucional para ejercer.

En un sentido integrador, la propuesta de comprensión de la Familia como sujeto político, está en correspondencia con la normativa jurídica de ser sujeto prevalente de derechos, y con la fundamentación ecosistémica para entenderla como un nuevo actor social, con protagonismo en la esfera gubernamental, en los movimientos sociales y en las políticas públicas. Este debate contemporáneo permea al Trabajo Social y contribuye al fortalecimiento de la identidad disciplinaria y a los procesos de renovación curricular y académica que requieren la apropiación de lenguajes especializados y de posicionar en otras disciplinas, como el Derecho, el saber propio.

\section{Referencias bibliográficas}

DE MARTINO, M., KRMPOTIC, C., Y MITJAVILA, M. (2008). El Trabajo Social en el campo socio-judicial: construcción socio histórica, modalidades, problemas y desafíos recientes en Argentina, Brasil y Uruguay. Revista Colombiana de Trabajo Social, (21).

MELANO, M. C. (2007). Aventuras y desventuras de los saberes técnicos. Revista de Trabajo Social (5), pp. 35-54.

Quintero VElÁsoueZ, A. (2007). Diccionario Especializado en Familia y Género. Buenos Aires: Lumen/Hvmanitas.

(2009). Pruebas Psicosociales en Derecho de Infancia, Adolescencia y Familia. Medellín: Universidad de Antioquia.

- (2010). El Trabajo Social en Colombia. Buenos Aires: Lumen/ Hvmanitas

REPÚBliCA DE COMBIA (1991). Constitución Política De Colombia. Recuperado el 12 de Marzo de 2010, de http://

7 asistentessocialesjudicialescolombia@yahoogroups.com 
wsp.presidencia.gov.co/Normativa/Documents/ ConstitucionPoliticaColombia_20100810.pdf

VATiCANO. Pontificio Consejo Para La Familia. (1983). Carta de los Derechos de la Familia. Recuperado el 10 de abril de 2010, de http://www.vatican.va/roman_curia/ pontifical_councils/family/documents/rc_pc_ family_doc_19831022_family-rights_sp.html 